\title{
Development of Field of View for Ground-based Optical Telescopes in Adaptive Optics
}

\author{
Xiaochun Zhong ${ }^{1,2, a}$, Shujuan Wang ${ }^{2, b}$, Zhiliang Huang ${ }^{3, c}$ \\ ${ }^{1}$ School of Physical Science and Technology, Southwest Jiaotong University, Chengdu, 610000, \\ China \\ ${ }^{2}$ Key Laboratory of Solar Activity, National Astronomical Observation, Chinese Academy of \\ Sciences, Beijing, 10000, China \\ ${ }^{2}$ School of Physical Electronic, University of Electronic Science and Technology of China, Chengdu, \\ 610000, China \\ aemail: zxc@swjtu.cn, bemail:wsj@bao.ac.cn, cemail:158555801@qq.com
}

Keywords: Adaptive Optics; Field of View; Tomography

\begin{abstract}
In this paper, we summarize the progress in the aspect of increasing field of view in adaptive optics. We present the isoplanatic challenge and the limits of conventional adaptive optics. Moreover, we briefly list the development of tomography and researches on the error of tomography in theory and experiment with the goal of reducing the limits imposed by anisoplanatism on conventional adaptive optics.
\end{abstract}

\section{Introduction}

Turbulence is distributed along the direction of propagation of light passing through the atmosphere. Atmospheric turbulence severely limits the angular resolution of ground-based telescopes. Adaptive optics (AO) can in principle allow a large telescope to achieve performance at its theoretical maximum (limited only by diffraction), by correcting in real time for the distortion of starlight by atmospheric turbulence [1]. AO compensates, in real-time, for the random fluctuations of wave-fronts induced by the turbulent atmosphere. The turbulent wave-front is detected by a wave-front sensor (WFS) and optically corrected by a deformable mirror (DM) located in a pupil conjugated plane. This compensation allows to record long exposure images with a resolution close to the diffraction limit. Nevertheless, the conventional AO approach is based on the assumption that the field of view (FOV) is smaller than the so-called isoplanatic field that is the field where the wave-fronts, coming from angularly separated points, can be considered as identical. In the visible, the isoplanatic field is about a few arcseconds [2]. If the FOV is greater than this field, the AO correction, which is maximum in the direction where the WFS measures the phase perturbation, is degraded as a function of angle [3]. A more promising solution to obtain a good correction in a large FOV is to consider a new AO approach through the use of several guide stars (GSs), i.e. several wave-front sensing directions, and several deformable mirrors (DMs) which are conjugated at different heights in the atmosphere. It is the concept of multi-conjugate AO (MCAO) [4]. With such a system, the turbulence effects are corrected not only in the telescope pupil but in the turbulence volume, hence increasing the isoplanatic field.

\section{Conventional Adaptive Optics}

The concept of AO was first proposed by American astronomer H. W. Babcock in 1953 [5]. Conventional adaptive optics systems use a single guide star to collect information regarding the line-integrated phase distortion from a single field angle. This limits the corrected field-of-view to a small isoplanatic patch around the guide star. Light coming from different field angles, however, travel through physically different regions in those turbulence layers that are removed from the entrance pupil of the system and are therefore not well corrected with a single guide star.

The turbulent wavefronts are detected by a WFS and optically corrected by a DM located in a 
pupil conjugated plane. As shown in Fig. 1.

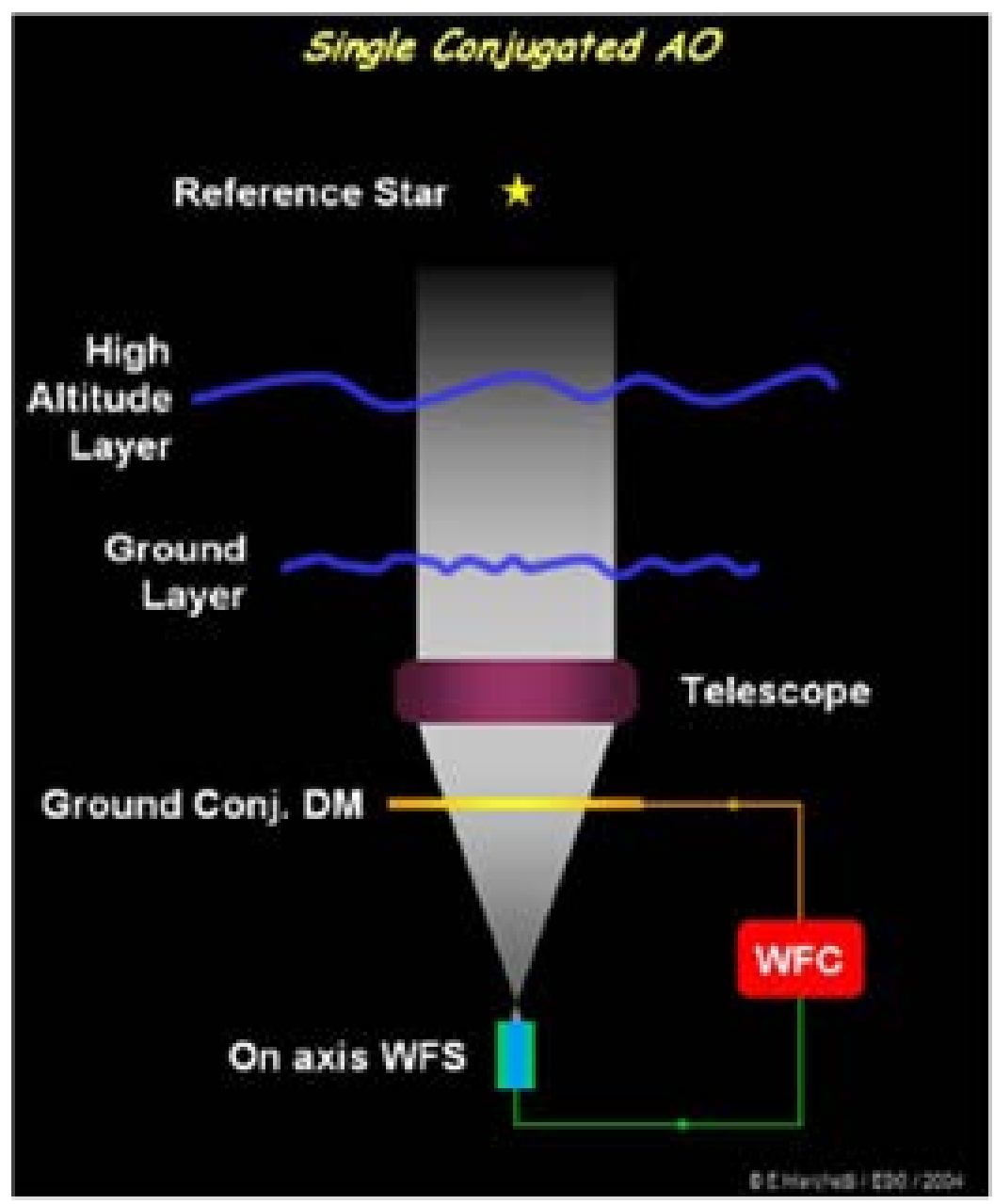

Fig.1. Conventional adaptive optics systems

Anisoplanatism is one of the most severe limitations on phase correction by adaptive optics. In the visible, the FOV of conventional AO is only about a few arcseconds. The small FOV over which AO provides diffraction limited resolution is the key barrier for the observations of extended target.

Angular anisoplanatism derives from the different regions in those turbulence layers through which the light from the guide star and the observed target travels, which leads to different phase aberrations in different direction of propagation of light from guide stars and the observed target passing through the atmosphere. Moreover, the WFS and DM are optically conjugated to a pupil plane in conventional adaptive optics system. Atmospheric turbulence is distributed along every direction of propagation of light passing through the atmosphere. As for extended observed target, only the area in the isoplanatic field could be imaged with a resolution close to the diffraction limit. Due to atmospheric anisoplanatism, the image of the field out of the isoplanatic field is badly degraded. It is the ineffectively correction of phase perturbations derived from 3-dimensional distribution of such turbulence with only one deformable mirror that lead to the small field of view.

\section{Multi-Conjugate Adaptive Optics}

MCAO was first proposed by J.M.Beckers in1988 as a technique to reduce the limits imposed by anisoplanatism on conventional AO [6]. MCAO is a further development of AO concept, where detection and correction is made by several Deformable Mirrors (DMs) and wave-front sensors (WFSs) conjugated to different altitudes. MCAO systems greatly reduce this limitation by collecting light from multiple guide stars at separate field angles. Through tomographic reconstruction of the resulting line-integrated phase distortion measurements from multiple field angles, the three-dimensional turbulence profile can be determined. In addition, multiple 
deformable mirrors are placed at conjugate altitudes to the turbulence layers in the atmosphere such that the individual turbulence layers can be corrected thereby greatly enhancing the corrected field-of-view. As shown in Fig. 2. Even without the use of multiple deformable mirrors, the tomographic reconstruction of the turbulence will be required to correct for focal anisoplanatism using laser guide stars in the next generation of extremely large telescopes.

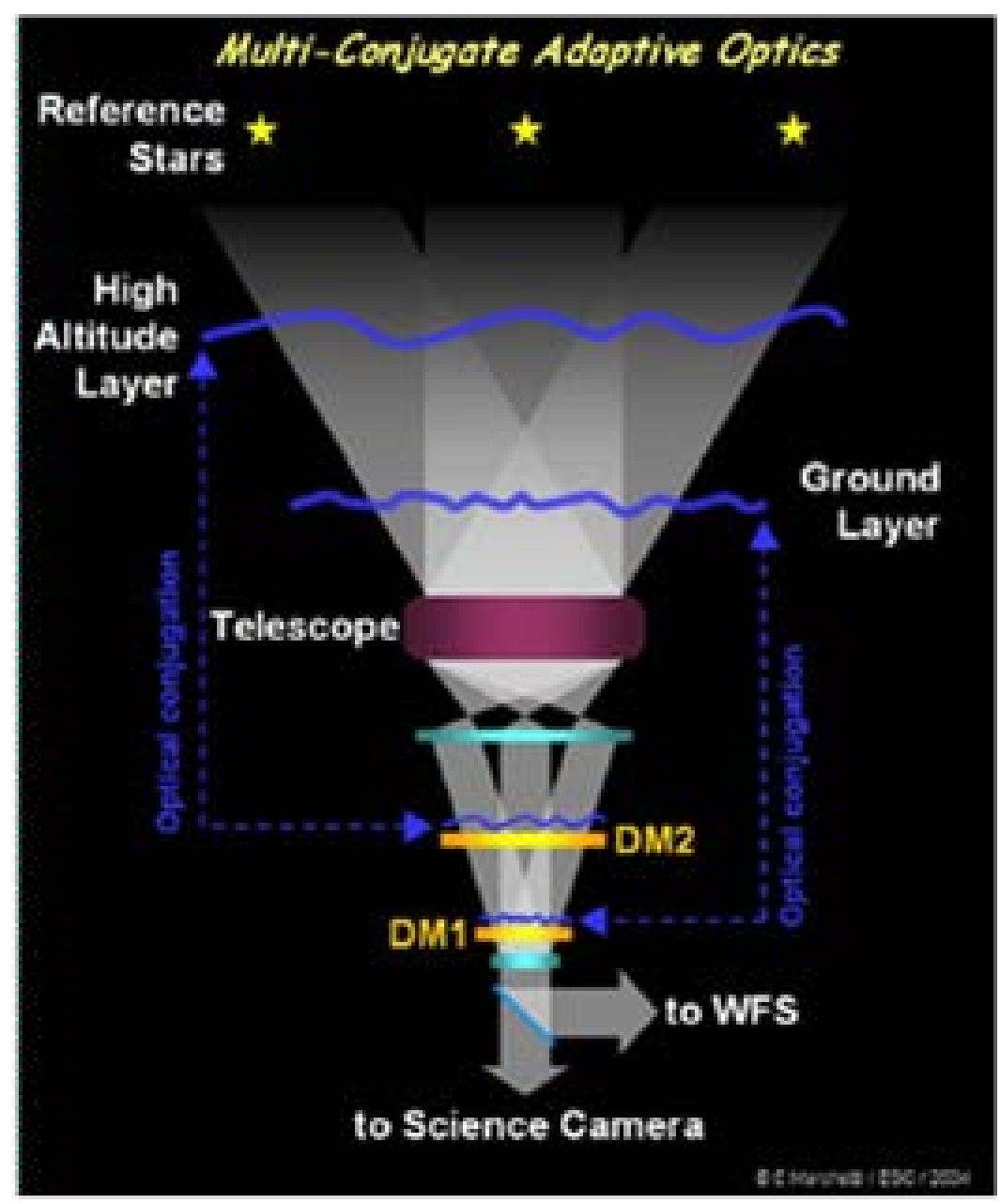

Fig.2. Multi-Conjugate Adaptive Optics system

After the proposition of the concept of MACO by J. M. Beckers, the most key challenge was how to retrieve 3-dimensional distribution and detection of atmospheric turbulence. Atmospheric tomography was proposed and analyzed by M. Tallon and R. Foy and was conceived as a method to measure the instantaneous 3-dimensional phase perturbations in the atmosphere, in order to get the control signals for MCAO [7]. Light from several Guide Stars (GSs) is used to probe the 3-dimensional perturbations, and retrieve the signals to control one or more DM can be derived directly from the information brought by GSs, without the need to reconstruct the whole turbulent volume, as show in Fig.3. The tomographic reconstruction of the turbulence is the core of MCAO technology. 


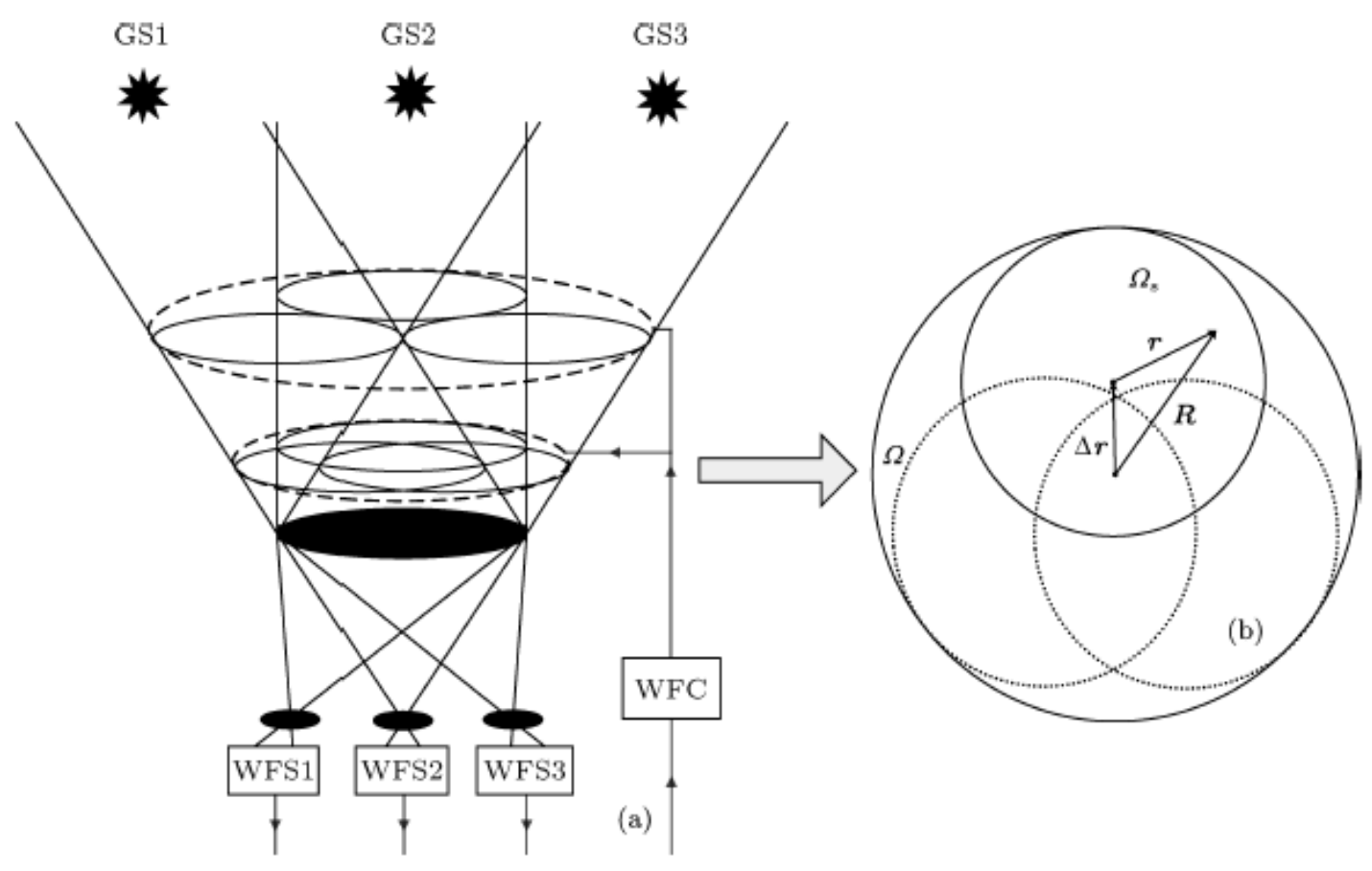

Fig.3. Atmospheric tomography

Zonal three-dimensional tomography sounds like one of the most promising technique to fully correct focal anisoplanatism. However, the technology of zonal tomography is based on some hypotheses which limit severely in the application of zonal tomography. Based on the same tomography concept, modal approach to the 3D-sensing, in contrast with the zonal approach was proposed by Roberto Ragazzoni, Enrico Marchetti, and Francois Rigaut. They analyzed several potential advantages of the modal approach [8]. Modal approach is a hot pot in solar adaptive optics which attracts more and more attentions now.

After the proposition of modal tomography, scientists have done a lot of research in theory and experiments. Through the experiment of observation of the space, it firstly demonstrated the correctness of the theory [9]. Two approaches are compared: the algorithm based on a Least Square (LS) approach and modal tomography. In the comparison, the concept of unseen modes was proposed. They concluded that the precision of modal tomography is limited in the detection of wave-fronts [10]. The frequency property of tomography was studied in the paper, which concluded that the error of the reconstruction algorithm is badly decreased by noises [11] . In the domestic, Zhaojun Yan has implemented simulation of the tomography algorithm in his $\mathrm{PhD}$ thesis [12]. Lanqiang Zhang has studied the origin of the error of modal tomography technology. The analysis shows that part Zernike model basis is used as a new basis of polynomial decomposition in model tomography, which leads to modal coupling error and aliasing error [13]. In his $\mathrm{PhD}$ thesis, Lanqiang Zhang designed a solar multi-conjugate adaptive optics system and studied solar MCAO and GLAO for the demand of high resolution observation of solar active region based on the modal tomography algorithm [14].

\section{Summary and Prospect}

MCAO promises to help offset the disadvantage of AO equipped ground based telescopes, which, compared to space observations, provide a small diffraction limited FOV (limited by the isoplanatic patch size). The sun is an ideal object for the development and application of MCAO, since the multiple wave-front measurements as a function of the FOV needed to perform atmospheric tomography can be performed using solar structure as wave-front sensing target. Solar AO has come a long way and is now close to becoming a mature technology that provides diffraction limited observations and thus will aid in solving many of the outstanding scientific problems of solar astronomy. 


\section{Acknowledgement}

This work was financially supported by Open Research Program of Key Laboratory of Solar Activity,National Astronomical Observatories, Chinese Academy of Sciences.

\section{References}

[1] Beckers, J. M. Adaptive optics for astronomy-principles, performance, and applications. Annu. Rev. Astron. Astrophys. 31, 13 \pm 62 (1993).

[2] D. L. Fried, Anisoplanatism in adaptive optics, [J]. Opt. Soc. Am. 72, pp. pp 52-61, Jan. 1982.

[3] F. Chassat, "Calcul du domaine d'isoplanétisme d'un système d'optique adaptative fonctionnant a travers la turbulence atmosphérique,” J. of Optics (Paris) 20(1), pp. 13-23, 1989.

[4] R. H. Dicke, "Phase-contrast detection of telescope seeing and their correction, Astron." J. 198(4), pp. 605-615, 1975.

[5] H. W. Babcock. The possibility of compensating astronomical seeing [J]. Publ. Astron Soc. Pac., 1953:65, 229-236.

[6] BECKERS J M. Multi-conjugate adaptive optics: Experiments in atmospheric tomography[J]. Proc of SPIE, 2000, 4007: 1056-1065.

[7] M. Tallon, R. Foy, Adaptive telescope with laser probe: isoplanatism and cone effect [J], Astron. Astrophys. 1990: 235, 549-557.

[8] R. Ragazzoni, E. Marchetti, F. Rigaut, Modal tomography for adaptive optics, Astron. Astrophys [J]. 1999: 342, L53-L56.

[9] Ragazzoni R, Marchetti E, Valente G, Adaptive-optics corrections available for the whole sky, Nature [J]. 2000:403 54.

[10] Fusco T, Conan J M, Michau V, Rousset G, Assemat F, Multi-Conjugate Adaptive Optics: Comparison of phase reconstruction approaches for large Field of View, Proc. SPIE [J]. 20014167 168.

[11] Tokovinin A, Viard E, Limiting precision of tomographic phase estimation, [J]. 2001 Opt. Soc. Am. A 18 873-882.

[12] Zhaojun Yan, Prediction Control and Multi-Conjugate Technologies for Adaptive Optics System [D], Institute of Optics and Electronics, Chinese Academy of Sciences in Chengdu, 2013.

[13] Lanqiang Zhang, NaiTing Gu, ChangHui Rao, Analysis of modal tomography for three-dimensional wave-front sensing of atmosphere turbulence, Chinese Physical Society [J]. 2013 6216.

[14] Lanqiang Zhang, Solar Muti-Conjugate Adaptive Optics for High Resolution Imaging [D], Institute of Optics and Electronics, Chinese Academy of Sciences in Chengdu, 2014. 